\title{
Fundamental Solution Method in a few MATLAB lines
}

\author{
A.D. Garnadi ${ }^{a, *}$ \\ a Department of Mathematics, Institut Pertanian Bogor, \\ Jl Meranti, Kampus Darmaga, Bogor 16680, \\ Indonesia
}

\begin{abstract}
We report a computational exposition to approximate solution of Laplace equation on the plane using Fundamental Solution Method. For a given boundary conditions at the boundary, we recover the approximate solution in the region. We demonstrate that fundamental solution method for the model leads to an unconstrained Quadratic Programming problem. To address the issue of ill-conditioning, we suggests to use SVD to overcome this issue. The implementation of the methods is using MATLAB.
\end{abstract}

Keywords: Fundamental Solution Method, 2-D Laplace Equations

\section{Notation}

$\begin{array}{ll}\xi \in \mathbb{R}^{N_{s} \times d} & \text { Sources } \\ x \in \mathbb{R}^{M_{D} \times d} & \text { (fixed) boundary coordinates } \\ f \in \mathbb{R}^{M_{D}} & \text { function values at } x \text { (pointwise) } \\ c \in \mathbb{R}^{N_{s}} & \text { coefficient for approximate function } \\ K \in \mathbb{R}^{M_{D} \times N_{s}} & \\ \tilde{u} \in \mathbb{R}^{M_{D}} & \text { approximate values at } x \text { (pointwise) } \\ \tilde{u}=K . c & \\ R=f-\tilde{u} & \end{array}$

\section{Model Problem}

Let $\Omega \subset \mathbb{R}$, be a bounded with boundary $\partial \Omega$. Consider the Laplace equation :

$$
\nabla^{2} u=0, \text { in } \Omega
$$

subject to boundary condition :

$$
u(x)=u_{D}(x), \text { on } \quad \partial \Omega
$$

\footnotetext{
${ }^{*}$ Corresponding author. E-mail: agah.garnadi@gmail.com
} 


\section{Fundamental Solution Method}

For domain $\Omega \subset R^{2}$, the fundamental solution :

$$
K(\mathbf{x} ; \xi)=-\frac{1}{2 \pi} \log |\mathbf{x}-\xi|, \mathbf{x} \in \Omega, \xi \notin \Omega,
$$

satisfy the equation (1.1). This function, might be used to solve BVP of Laplace Equation (1.2). The techniques is via approximation :

$$
\tilde{u}_{N_{s}}(\mathbf{x})=\sum_{i=0}^{N_{s}} c_{i} K\left(\mathbf{x} ; \xi_{i}\right),\left\{\xi_{i} ; i=1 \cdots N\right\} \not \subset \Omega .
$$

Through least squares fit to the prescribed boundary values, we will recover the coefficient weights of the approximant. Let $\left\{x_{i}^{D} ; 1 \leq i \leq M_{D}\right\} \neq \emptyset$, denotes points sampled from Dirichlet boundary. Residual at each point :

$$
R_{D}(j)=u_{D}\left(x_{j}^{D}\right)=\sum_{i=0}^{N_{s}} c_{i} K\left(\mathbf{x}_{j} ; \xi_{i}\right), 1 \leq j \leq M_{D} .
$$

Rewriting the problem in a matrix format, we ends up to the following form :

$$
\mathbf{R}_{D}=\mathbf{u}_{D}-K_{D} \quad \mathbf{c}
$$

for Dirichlet residual.

Defining least-squares functional:

$$
F=\frac{1}{2}\left(\mathbf{R}_{D}^{t} \mathbf{R}_{D}\right)
$$

For details of this method and the range of its application, we refer to [1] [2] and references therein.

\section{Data representation of domain $\Omega$}

The domain $\Omega$ can be described geometrically based on points of the boundary $\partial \Omega$. The points, herewith we called it nodes lies at the Dirichlet boundary, $\Gamma_{D}$. Where $\partial \Omega$.

Through *.dat files, MATLAB able to support reading ASCII data format. In figure (One), shows nodes on the boundary of $\Omega$.

In our implementation, the node at Dirichlet boundary, are given in dirichlet.dat contains in each row the node numbers of corresponding coordinates with Dirichlet type boundary :

\begin{tabular}{|clll}
\hline \multicolumn{3}{|c}{ Dirichlet_node_number } & X- \\
\multicolumn{3}{c}{ For example : } \\
1 & 0.0 & 0.0 & 0.0 \\
2 & 0.0 & 0.25 & 0.0 \\
3 & 0.0 & 0.5 & 0.0 \\
4 & 0.0 & 0.75 & 0.0 \\
5 & 0.0 & 1.0 & 0.0 \\
6 & 0.25 & 1.0 & 0.25 \\
7 & 0.5 & 1.0 & 0.5 \\
8 & 0.75 & 1.0 & 0.75
\end{tabular}




$\begin{array}{llll}9 & 1.0 & 1.0 & 1.0 \\ 10 & 1.0 & 0.0 & 1.0 \\ 11 & 1.0 & 0.25 & 1.0 \\ 12 & 1.0 & 0.5 & 1.0 \\ 13 & 1.0 & 0.75 & 1.0\end{array}$

From this data we extracts variable $\mathbf{x}_{-} \mathbf{D} \in \mathbb{R}^{M_{d} \times 2}$ and variable $\mathbf{f} \_\mathbf{D} \in \mathbb{R}^{M_{D}}$, where $M_{D}$ is total number of dirichlet nodes, in our example above $M_{D}=10$.

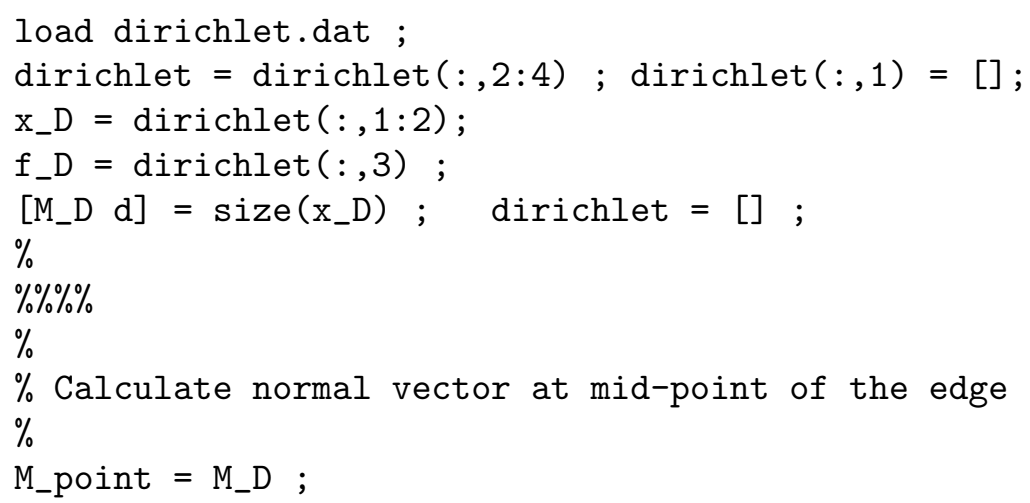

Based on coordinates of Dirichlet nodes and edges mid-point, we generate sources point. We arranged the sources positioned at a fixed radius circle with centre at the boundary coordinates centre of mass. The sources coordinates are generated by a call of MATLAB scripts in the next section.

To obtain the weights $\mathbf{c}$ we call the solver:

[C_star, Xi] = solvelap (x_D,f_D ) ;

which the MATLAB scripts described in the next section.

\section{Computation and Visualization}

With these description of coordinates of Dirichlet boundary, the function evaluation of the matrices entry can be evaluated directly, i.e. :

$$
\left(K_{D}\right)_{i j}=K\left(\mathbf{x}_{j} ; \xi_{i}\right), 1 \leq i \leq N_{s} ; 1 \leq j \leq M_{D}
$$

Defininng the matrix $\mathrm{K}$ as:

$$
K=\left[K_{D}\right]
$$

and functions value at boundary in a contiguous column vector :

$$
f=\left[u_{D}\right]
$$

The problem is the following least squares problem :

$$
\min _{\mathbf{c}}\left\{\frac{1}{2}\|K \mathbf{c}-f\|^{2}\right\}
$$

The least-squares solution is: 


$$
c^{*}:=\left(K^{t} K\right)^{\dagger} K^{t} f
$$

where $\left(K^{t} K\right)^{\dagger}$ denoting the generalized inverse of $\left(K^{t} K\right)$

These stages are implemented in MATLAB functions scripts as follows:

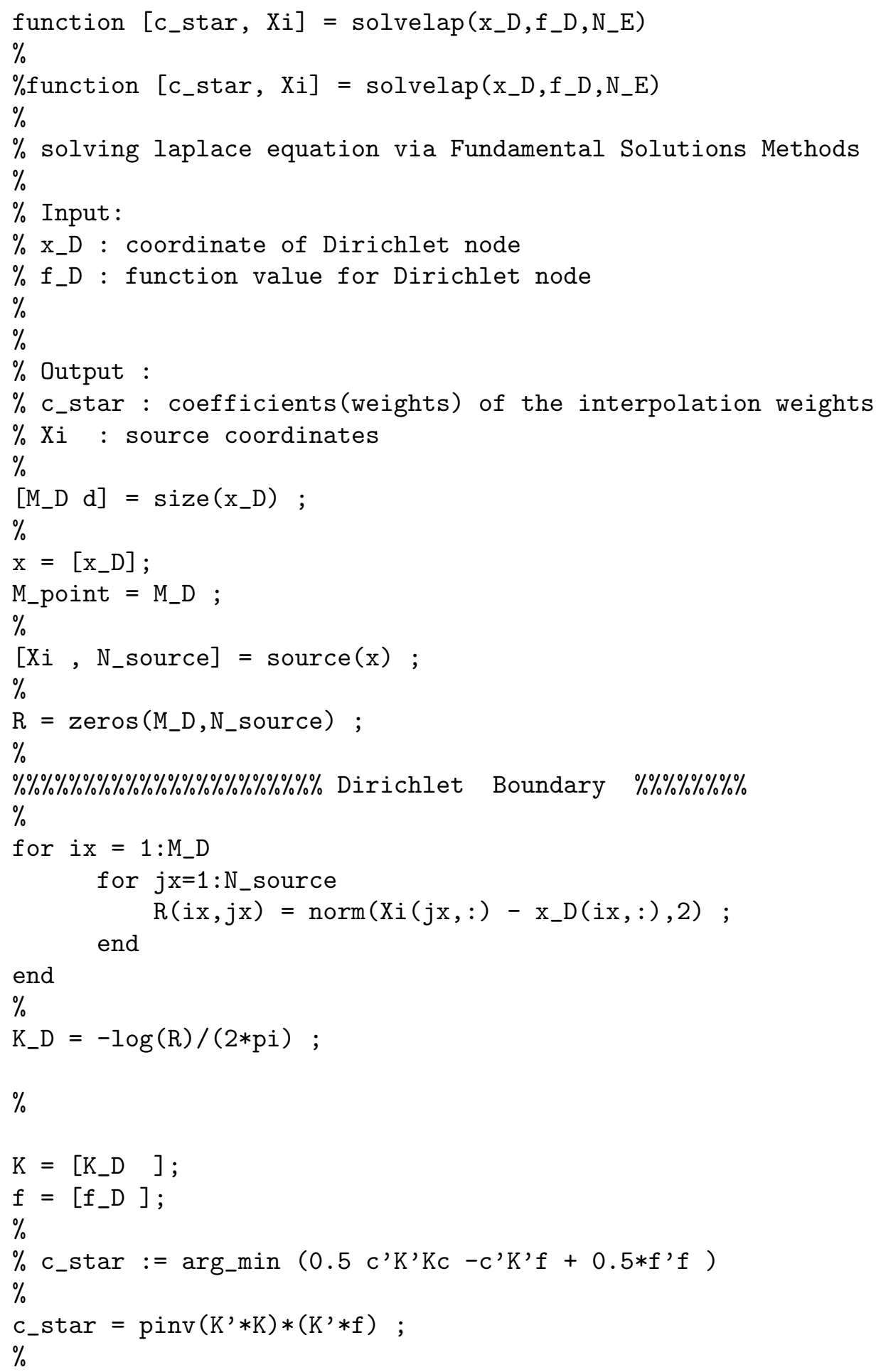


Visualisation, is depends on provided geometry. So provided points of interest, we evaluate the aproximate function on the points of interest. In this case we needs kernel evaluation :

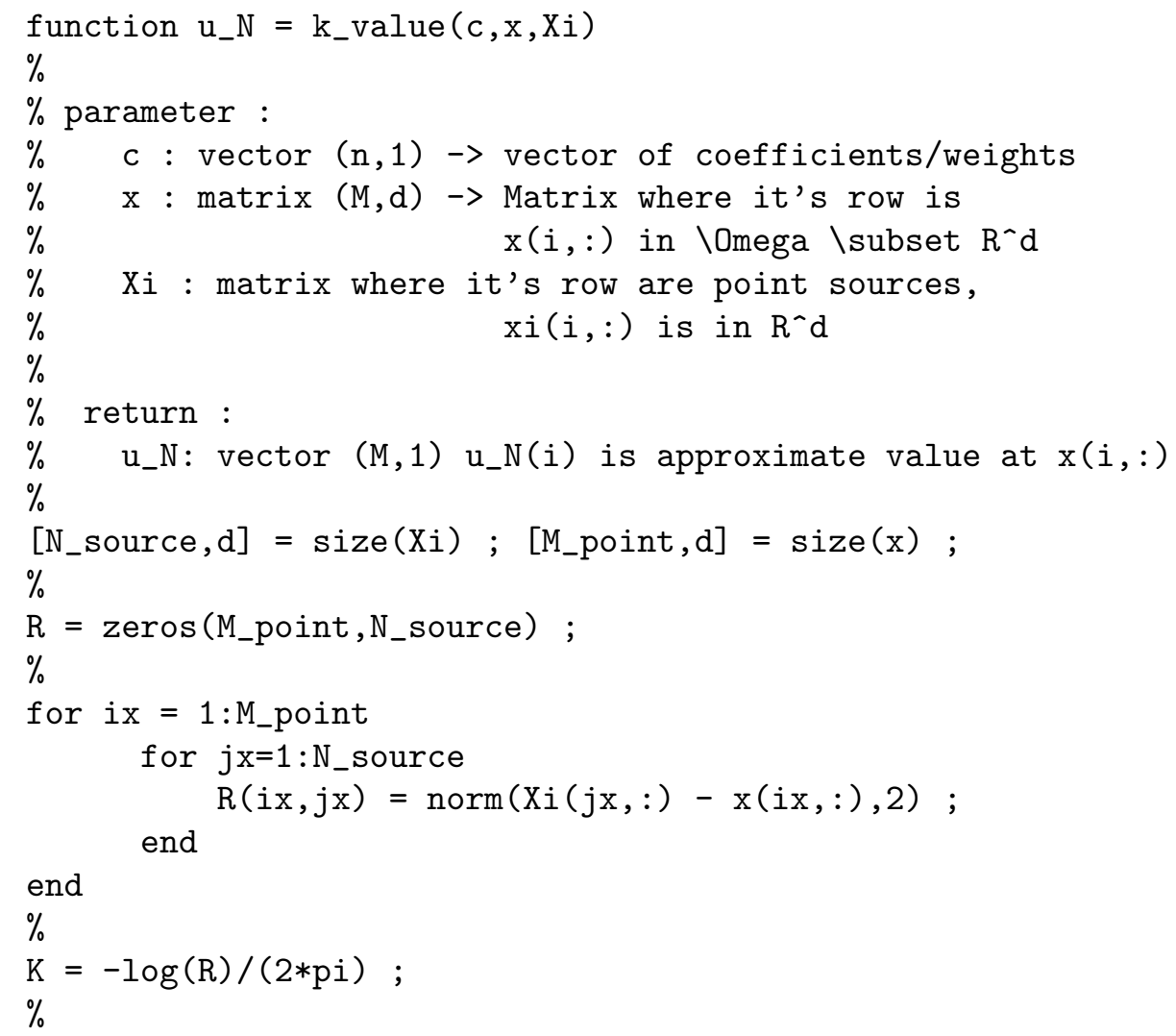

For our example above, the interior point bounded in by the boundary is of interest. Then we evaluate the approximate value at its corresponding point througs another MATLAB script functions.

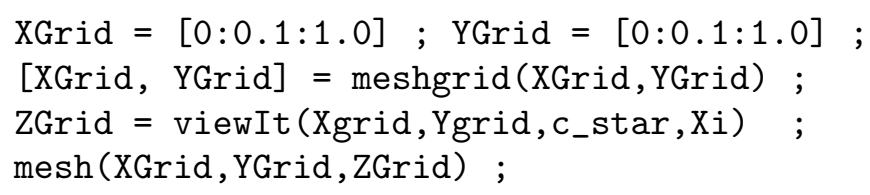

The utility evaluation function scripts, are as follows:

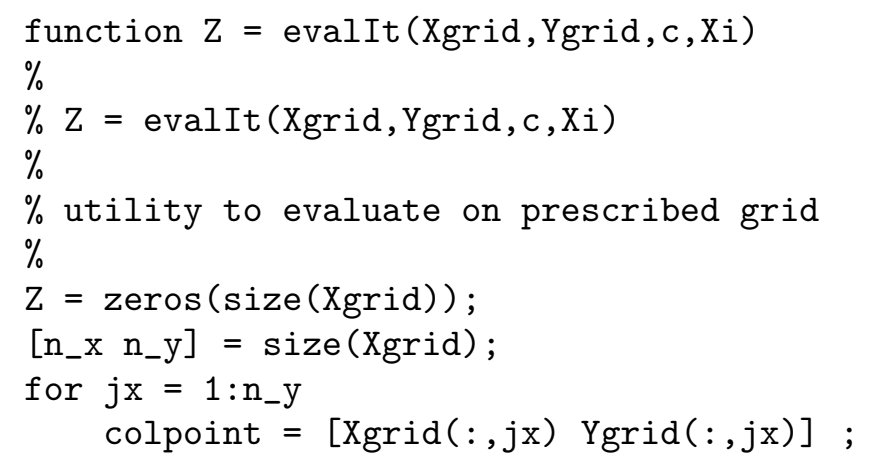


end

$$
\mathrm{Z}(:, \mathrm{jx})=\mathrm{k}_{-} \text {value }(\mathrm{c}, \text { colpoint, } \mathrm{Xi}) ;
$$

\section{References}

[1] G. Fairweather, Fundamental Solution Methods, CMSE97 Proceedings, Bandung.

[2] A. Karageorghis \& G. Fairweather, 1998, The Method of Fundamental Solutions for Elliptic boundary problems, Advances in Computational Mathematics, 9, 69-95 